\title{
Dynamical Systems and Network Flows: Traffic Flow Problem on Multi-lane Intersections (Economic Analysis)
}

\author{
Tarekegn Mitiku Agajie \\ Department of Mathematics, Arba Minch University, Arba Minch, Ethiopia
}

Email address:

tarekegnmitiku@gmail.com

To cite this article:

Tarekegn Mitiku Agajie. Dynamical Systems and Network Flows: Traffic Flow Problem on Multi-lane Intersections (Economic Analysis). Applied and Computational Mathematics. Vol. 4, No. 4, 2015, pp. 321-330. doi: 10.11648/j.acm.20150404.20

\begin{abstract}
Nowadays due to rapid population growth and hence increasing demand for transportation, traffic congestion at road intersections become a serious problem for developed as well as developing countries. Traffic congestion causes considerable costs due to unproductive time losses, extra fuel consumption, accidents and also has a negative impact on the environment such as air pollution, noise and stress. Thus, economic analysis of multi-lane intersections and improvement alternatives take account of vehicles cost of fuel consumption and time costs incurred by users of the road junctions. The objective of this study was about determining average waiting time of vehicles and estimating cost incurred due to delay at unsignalized double lane roundabouts and signalized cross intersections. In this study MMAS Cellular Automata model and Poisson queuing model were used. The study tried to calculate the average waiting time (delay) of vehicles in the system (in queue plus in service) at both types of road intersections. The study was tried to quantify vehicles waiting time at both types of road intersections (cost incurred due to delay); that is cost of time lost for passengers and cost of extra fuel consumed by vehicles. Based on the findings of the study, that is based on time and fuel lost (though other factors are not included), signalized cross intersections are better than roundabouts to minimize traffic congestion problem at road junctions.
\end{abstract}

Keywords: Roundabout, Cross Intersection, Poisson Queuing Model, Average Waiting Time, Number of Vehicles in the System, Cost of Time Lost, Cost of Extra Fuel Consumed

\section{Introduction}

\subsection{Background of the Study}

For more than three decades modern roundabouts have been used throughout the world as a junction control device. Addis Ababa also has its share of roundabouts. There are three legs; four legs; five legs and six legs roundabout in Addis Ababa. The performance of roundabouts during offpeak periods is particularly good in contrast to other intersection forms, typically with very low average delays [4] When we deal about roundabouts in this thesis, we mean Multi-lane (double lane) unsignalized roundabouts because single-lane roundabouts proved inadequate [1]. Traffic congestion is frequently observed

on road intersections during rush hours in Addis Ababa city. This imposes economic impacts on the urban dwellers in many aspects. To precisely identify the magnitude of economic impact on urban, it needs deep investigation in terms of economic analysis of roundabouts and signalized intersections, to propose which is more economical for use.
Even though a wide range of traffic flow theories and models have been developed, still congestion is one of the main societal and economical problems related to transportation throughout the world. As an example, the 2009 Urban Mobility Report of Texas Transportation Institute [7] states that, congestion caused urban Americans to travel 4.2 billion hours more and to purchase an extra 2.8 billion gallons of fuel for a congestion cost of $\$ 87.2$ billion.

Though economic analysis is a clear effect which is a major factor to select the best possible form of road intersection, there is no comprehensive study made regarding the economic benefit of the choice of intersection, to the knowledge of the author. Due to rapid population growth and hence increasing demand for transportation, traffic congestion at these road intersections become a serious problem for developed as well as developing countries [2]. Traffic congestion causes considerable costs due to unproductive time losses, extra fuel consumption, accidents and also has a negative impact on the environment such as air pollution, noise and stress. Thus, economic analysis of multilane intersections and improvement alternatives take account 
of vehicles cost of fuel consumption and time costs incurred by users of the facility.

\subsection{Statement of the Problem}

Traffic congestion is not expected to disappear in the near future in road intersections. However, innovative measures are needed to alleviate the situation. In many cases where traffic demand far exceeds the capacity, the intersections can be inefficient. Lane addition is one resort available to traffic engineers and has been used extensively to increase the capacity of the intersections. Although adding turning or through lanes may provide a short term relief, that solution is often infeasible because it may simply be too expensive to continuously widen the existing intersection.

Now a days, it is common to see traffic congestion at junctions in Addis Ababa particularly during rush times. For example, at Torhayloch, Urael, Mexico, Imperial,etc intersection areas large number of vehicles form long queue. Hence, traffic police need to intervene in situations to regulate the traffic flow. Otherwise, it would be practically impossible to have normal traffic flows, especially at roundabout junctions, which is more dependent on driver behavior and balanced traffic flow between the approaches [1]. This problem may continue and it may worsen in the future due to the rapid growth of population and vehicles number in Addis Ababa. In parallel to the increment in the number of population in the city, the problem of congestion is also increasing from time to time. This may hamper the economic growth of urban dwellers unless appropriate remedial action is taken based on research results. Clearly the consequence of queued vehicles at the intersection causes a delay for users to reach their home or work place, increase in fuel consumption and pollution of the environment.

To determine simulated results of operational performance for roundabouts and signalized cross intersections, Multistream Minimum Acceptable Space (MMAS) Cellular Automata (CA) model based on the Minimum Acceptable sPace (MAP) method was used [3]. Performance measurements for roundabouts and signalized cross intersections include throughput (the number of vehicles that can navigate a roundabout or cross intersection), capacity (the maximum number of vehicles that can pass through a roundabout from a given road), queue length, delay etc. In [3]., except delay (average waiting time of vehicles at road intersections) most of the performance measures mentioned above obtained using Multi-stream Minimum Acceptable Space (MMAS) CA model. However, the research done in [3] focused only on simulating most of operational performance for both types of road intersections.

So, to fill the gap in this thesis, the same model is intended to be used to find out simulated result of average waiting time which was the most difficult to estimate but the most important measure of operational performance for our cost analysis. By using the aforementioned performance measures (throughput, queue length, and so on) as input, we would calculate average waiting time of vehicles combining with the above model and other model called Poisson queuing model as we briefly discussed in section 2 . Once we determine the average waiting time (delay) of vehicles at both road intersections, we quantify it to: cost of lost time for passengers' and cost of extra fuel consumed for vehicles at both road junctions. Therefore, a thorough analysis should be done and comparison of the performance of the kind of intersection are to be taken to come up with the appropriate solution.

\subsection{Objectives of the Study}

\subsubsection{General Objective}

The study aims to give the economic analysis of double lane roundabouts and signalized intersections.

\subsubsection{Specific Objectives}

The principal objectives of this study are to:

- Compute and compare the average waiting time and number of vehicles in the system (due to delay) for unsignalized double lane roundabout and signalized double lane cross intersection.

- Calculate and quantify the difference in waiting time (delay) of vehicles at the road junctions.

- Estimate average cost of extra fuel consumed by vehicles at the road junctions

- Estimate average cost of passengers and drivers time lost due to congestion at the road junctions

\section{Models}

\subsection{Cellular Automata Model}

Cellular Automata (CA) model is used to study dynamical systems in which both time and space are considered as discrete variables, and physical quantities take on a finite set of discrete values. i.e., cellular automaton model use integer variables to describe the dynamical properties of the system. It consists of a regular grid of cells, each of which can be in a finite number of possible states, updated synchronously in discrete time steps. The state of a cell is determined by the previous states of surrounding neighborhood of the cell. For traffic model, the road is divided into a constant length $\Delta x$ representing a single cell and the time is divided to in steps of $\Delta t$. Each grid of cells (road section) can either be occupied by a vehicle or empty and the dynamics are given by update rules discussed below.

Most of the studies ([5],[6],[7],[8]) show that each cell is occupied by one small vehicle (SV) corresponding to a standard car of length $\leq 7.5$ meters, medium vehicles (MV) are taken to be double the length of a standard car and understood to be a bus or equivalent vehicle i.e., two cells are required for one $\mathrm{MV}$ and three cells are taken for very large vehicle (LV). For example, for small vehicles (SV) setting $\Delta x=7.5$ leads to a model where one vehicle always occupies exactly one section of the road with in one second time and a velocity of passing 5 cells corresponds to $5 \frac{\Delta x}{\Delta t}=$ $135 \frac{\mathrm{km}}{\mathrm{h}}$, which is set to be the maximum velocity a driver wants to drive. However, in such a model, smallest possible 
acceleration would be $\frac{\Delta x}{\Delta t^{2}}=7.5 \frac{\mathrm{m}}{\mathrm{s}^{2}}$ which is unrealistic. Therefore, many modern CA models use a finer spatial discretization, for example $\Delta x=1.5 \mathrm{~m}$, leading to a smallest possible acceleration of $1.5 \mathrm{~m} / \mathrm{s}^{2}$.

However, in this study the approximated single cell is about 4.17 meters that is the average length of vehicles in the study area and assumed average speed of a car within a roundabout is $8.33 \mathrm{~m} / \mathrm{s}$ traversing a cell within 0.5 seconds [3]. Although cellular automaton models lack the accuracy of the time-continuous car-following models, they still have the ability to reproduce a wide range of traffic phenomena. Due to the simplicity of the models, they are numerically very efficient and can be used to simulate large road networks in real time or even faster.

\subsection{Update Rule for the Roads}

The update rule can be expressed in terms of the sequential cells. If there is a vacant cell in front of the cell occupied by a vehicle, the vehicle will move forward one cell in the current time step. If the cell in front is occupied too, the vehicle will stop there in that time step. Figure 1 shows the graphical representation for this rule. The state $C_{n}^{t+1}$ of the $n^{t h}$ cell in the next time step is decided by the states of its own cell $C_{n}^{t}$, the state of the cell in front and the cell behind in this time step, where $t$ and $n$ express time and position sequence respectively. The states can only be 0 or 1 , where 0 means the cell is vacant and 1 means a car occupies the cell.

The update rule can be described as follows:

$$
\begin{aligned}
& \text { If } C_{n}^{t}=1 \text { and } C_{n+1}^{t}=0, \text { then } C_{n}^{t+1}=0 \& C_{n+1}^{t+1}=1 \\
& \text { If } C_{n}^{t}=0 \text { and } C_{n+1}^{t}=0, \text { then } C_{n+1}^{t+1}=0 . \\
& \text { If } C_{n}^{t}=1 \text { and } C_{n+1}^{t}=1, \text { then } C_{n+1}^{t+1}=1 .
\end{aligned}
$$

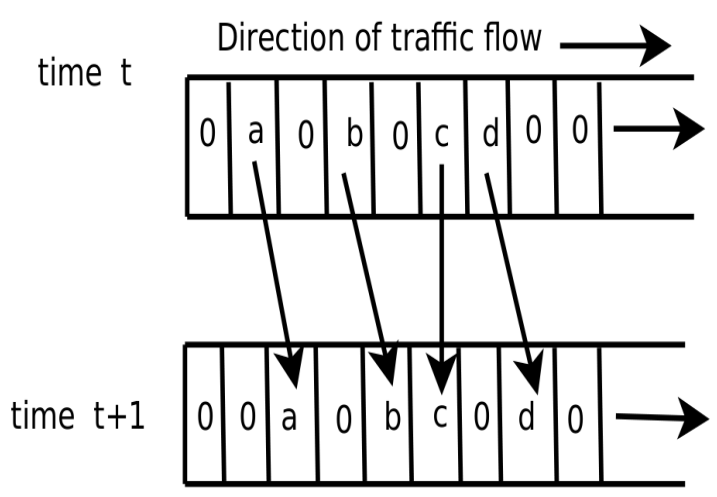

Figure 1. update rule on the roundabout.

\subsection{Multi-stream Minimum Acceptable Space (MMAS)}

Multi-stream Minimum Acceptable Space (MMAS) Cellular Automata (CA) model is used to investigate traffic flow at multi-lane intersections. The model considers the give-way rule appropriately and describes the details of vehicle movements and interactions from different entry roads. It was based on the Minimum Acceptable sPace (MAP) method as dealt by Wang and Ruskin [1].. MAP can be designed to describe both heterogeneous and inconsistent driver behavior, as well as random interaction between individual vehicles in cross traffic flow, independent of headway distribution considerations. As such, the MAP method can be applied to most features of traffic flow and has been used for road intersections such as roundabouts and cross intersections.

The performance indicators of unsignalized multi-lane roundabouts like throughput, capacity and queue length can be simulated using the Minimum Acceptable sPace (MAP) method [1].. In the MAP method, driver behavior is categorized into four groups: radical, urgent, moderate and conservative. If a driver accepts a 3-cell space as the MAP and enters the roundabout, behavior is designated as moderate. Radical behavior requires one cell space. This may cause delay in the coming vehicles on the roundabout and may generate gridlock [1]. A 2-cell space corresponds to urgent behavior, which may be the result of misjudgment, over confidence in vehicle acceleration, and bad driving habits that also cause delay like radical behavior of drivers. Conservative behavior corresponds to MAP with greater than or equal to 4 cells gap which is clearly considered as abnormal especially at low arrival rates. MAP and MMAS are a combination of both spatial and temporal conditions. For example, MAP is determined by the number of cells needed to represent driver behavior of the various types as discussed above, when entering a road intersections. In the model a vehicle can move forward one cell in each time step on a roundabout. Therefore, the number of cells of MAP correspond to the headway between the two circulating vehicles on a roundabout. On the other hand, the space for a vehicle to enter a roundabout must have clearance of specific cells for entry; otherwise the entering vehicle must wait to join different roundabout lanes. Therefore, MAP and MMAS describe both temporal and spatial behavior of drivers at roundabouts.

The operational performance of the road intersections (throughput, arrival rate, queue length etc) could be used to determine average waiting time (delay) of vehicles at each road intersection. But determining the average waiting time is the most difficult task and hence in the next section we derive how to determine the average waiting time of vehicles in the system (vehicles in queue plus in service).

\subsection{Poisson Queuing Model}

Queuing theory is a mathematical study of queues having a waiting line especially of persons or vehicles wait for services. Queuing theory helps to provide decision or guideline on system capacity. Studying queue theory is a difficult task due to probabilistic nature of the problem. Among many statistical queue models Poisson distribution was employed to simulate the steady state operational performance according to equation (1). Because in Poisson distribution the random variable, in our case the number of vehicles, is a discrete variable.

It is impossible to exactly forecast the time when vehicles arrive at the entrance road of intersections because the vehicles' arrival to such road junctions is random in nature. But it is possible to approximate their distribution using 
Poisson queuing model to determine the probability distribution of vehicles' arrival that have random distribution of vehicles. For a Poisson queuing model, the probability of $r$ arrivals in $t$ time has the following density function:

$$
P(r)=\frac{(\lambda t)^{r} e^{-\lambda t}}{r !}
$$

Where:

$P(r)=$ Probability of having $r$ vehicles arrived in time $t$.

$\lambda=$ average vehicle arrival rate in vehicles per unit time,

$r=$ Number of vehicles expected to arrive during time $t$,

$t=$ Time interval in which $r$ vehicles are expected to arrive.

Hence at different arrival rates $(\lambda)$, we need to find the probability of $0,1,2, \ldots, v-1$ or $v$ arrivals by using equation (1).

\subsubsection{Derivation of Steady State Probabilities}

The system is said to be at steady state when the rate of arrivals of vehicles is less than the rate of service. Arrival of vehicles at the entrance of road intersections is completely independent of time. Though the size of the queue fluctuates in steady state the statistical behavior of Poisson distribution of the queue remains steady. Therefore, the probability of finding a particular length of the queue at any time will be the same and hence a steady state condition is achieved.

\subsubsection{Now Let Us Define Some Terminologies}

$n=$ Total number of vehicles in the system; i.e., number of vehicles in queue plus in service.

$\lambda_{n}=$ Arrival rate given $n$ vehicles in the system; i.e., arrival rate is the rate at which vehicles are expected to arrive at the road intersections within a specified period of time, assuming that there are $n$ vehicles in the system.

$\mu_{n}=$ Service rate given $n$ vehicles in the system; i.e., service rate specifies the average number of vehicles that can be serviced during a time period.

$P_{n}=$ Steady state probability of $n$ vehicles in the system.

Once we derive $P_{n}$ as a function of $\lambda_{n}$ and $\mu_{n}$, then steady state measures of performance like average waiting time in the queue, average waiting time in the system, average waiting time in the service and so on could be easily determined. The probabilities $P_{n}$ are determined by using the transition-rate diagram shown in Figure 2. The queuing system is said to be in state $n$ when the number of vehicles in the system is $\mathrm{n}$.

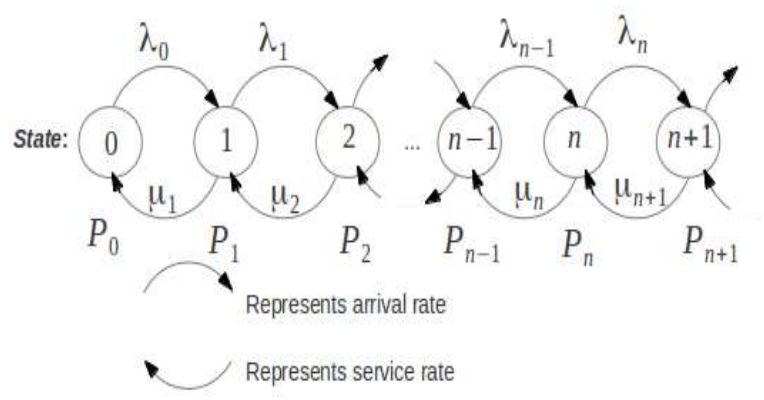

Figure 2. Poisson queue transition-rate diagram.
For example, state 0 means no vehicle in the system. So, state 0 can only change to state 1 when an arrival occurs at the rate $\lambda_{0}$ and $\mu_{0}$ is undefined because no departures occur if the system is empty. Under steady state conditions, for any state $n$ the expected rates of flow into and out of this state must be equal. The equation expressing this principle is called the balance equation for state $\mathrm{n}$ as shown in equation (2). After constructing the balance equations for all the states in terms of the unknown $P_{n}$ probabilities, we can solve this system of equations to find these probabilities. From Figure 2, the balance equation is solved recursively associated with $(n=0,1,2, \ldots)$. The arrows in Figure 1 shows the only possible transitions in the state of the system within a sufficiently small interval of time, and the entry for each arrow gives the mean rate either entering or leaving for that transition.

To make clear the balance equation, by using example, consider state 0 from Figure 2. The process enters this state only from state 1 . Thus, the steady state probability of being in state $1\left(P_{1}\right)$ represents the proportion of time that it would be possible for the process to enter state 0 . Given that the process is in state 1 , the mean rate of entering state 0 is $\mu_{1}$. From any other state, this mean rate is 0 . Therefore, the overall mean rate at which the process leaves its current state to enter state 0 , the mean entering rate is: $\mu_{1} P_{1}+$ $0\left(1-P_{1}\right)=\mu_{1} P_{1}$. By the same reason, the mean leaving rate is $\lambda_{0} P_{0}$. Hence, the balance equation for state 0 could be $\mu_{1} P_{1}=\lambda_{0} P_{0}$, solving this in terms of $P_{1}$ yields $P_{1}=$ $\frac{\lambda_{0}}{\mu_{1}} P_{0}$.

In the same manner for state 1 by using Figure 2 we have $\lambda_{0} P_{0}+\lambda_{2} P_{2}=\left(\lambda_{1}+\mu_{1}\right) P_{1}$, solving in terms of $P_{2}$ and substituting $P_{1}=\frac{\lambda_{0}}{\mu_{1}} P_{0}$ and after simplification, we have $P_{2}=\frac{\lambda_{1} \lambda_{0}}{\mu_{2} \mu_{1}} P_{0}$. Therefore, the remaining states can be determined (for $n=2,3, \ldots$ ) in the same way using the transition rate diagram (Figure 2).

In general, for $n>0$, state $n$ can change only to two possible states:

1. $n-1$ when a departure occurs at the rate $\mu_{n}$

2. $n+1$ when an arrival occurs at the rate $\lambda_{n}$

In conclusion, based on the fact that state $n$ can be changed to states $n-1$ and $n+1$ only, we have:

$$
\begin{aligned}
& \text { expected rate of flow in to state } n= \\
& \text { expected rate of flow out of state } n
\end{aligned}
$$

$$
\begin{array}{r}
\text { State 0: } \mu_{1} P_{1}=\lambda_{0} P_{0} \Rightarrow P_{1}=\frac{\lambda_{0}}{\mu_{1}} P_{0} \\
\text { State 1: } \mu_{0} P_{0}+\mu_{2} P_{2}=\left(\lambda_{1}+\mu_{1}\right) P_{1} \\
\Rightarrow P_{2}=\frac{\lambda_{1} \lambda_{0}}{\mu_{2} \mu_{1}} P_{0}
\end{array}
$$

State 2: $P_{3}=\frac{\lambda_{2} \lambda_{1} \lambda_{0}}{\mu_{3} \mu_{2} \mu_{1}} P_{0}$, then by induction we get:

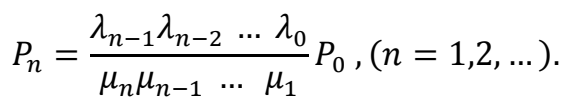




$$
\Rightarrow P_{n}=\left(\frac{\lambda}{\mu}\right)^{n} P_{0}(n=1,2, \ldots) .
$$

Let $\frac{\lambda}{\mu}=\rho$. Here, $\rho$ is called utilization factor.

$\Rightarrow P_{n}=\rho^{n} P_{0}(n=1,2, \ldots)$. and $P_{0}$ can be determined from $\sum_{n=0}^{\infty} P_{n}=\sum_{n=0}^{\infty} \rho^{n} P_{0}=1$

$$
\begin{gathered}
\Rightarrow P_{0}=1-\rho^{n} \\
P_{n}=\rho^{n}(1-\rho)
\end{gathered}
$$

\subsection{Derivation of Steady State Performance Measures}

Using the steady state probability discussed in section 2.2.1, we can obtain some performance measures of the system (in our case the system is the road intersection) that can be defined in a queuing system as follows:

$L_{s}=$ average number of vehicles in the system

$L_{q}=$ average number of vehicles in the queue

$L_{r}=$ average number of vehicles in service

$W_{s}=$ average waiting time in the system

$W_{q}=$ average waiting time in the queue

$W_{r}=$ average service time

Note: all these measures of performance have positive values including the arrival rate, inter-arrival rate, service rate and service time.

We now use the steady-state probability distribution given in equation (2) to determine several quantities of interest. For example, assuming that the steady state has been reached, by definition the average number of vehicles present in the queuing system $\left(L_{s}\right)$ is given by

$$
\begin{gathered}
L_{s}=\sum_{n=0}^{\infty} n P_{n} \Rightarrow L_{s}=\sum_{n=0}^{\infty} n \rho^{n}(1-\rho) \\
L_{s}=(1-\rho) \sum_{n=0}^{\infty} n \rho^{n}
\end{gathered}
$$

As clearly seen in equation (4) queue increases infinitely if $\rho>1$ i.e., $\mu<\lambda$. In other words, if the number of vehicles that can be served per period of time is less than the average number of vehicles arriving, the waiting line grows infinitely. Even queue can occur when $\lambda<\mu$ in stochastic case because arrival of vehicles to road intersections are random. In fact, $\rho<1$ or $\lambda<\mu$ is one of the requirements for the study of queuing theory and for $\lambda<\mu$, steady state can be achieved.

Defining:

$$
\begin{gathered}
S=\sum_{n=1}^{\infty} n \rho^{n} \\
\Rightarrow S=\rho+2 \rho^{2}+3 \rho^{3}+4 \rho^{4}+5 \rho^{5}+\cdots(*)
\end{gathered}
$$

Multiply equation $(*)$ by $\rho$ yields

$$
\rho S=\rho^{2}+2 \rho^{3}+3 \rho^{4}+4 \rho^{5}+\cdots(* *)
$$

Subtracting equation $(* *)$ from equation $(*)$ gives:

$$
\begin{gathered}
S-\rho S=\rho\left(1+\rho+\rho^{2}+\rho^{3}+\rho^{4}+\rho^{5}+\cdots\right) \\
\Rightarrow S(1-\rho)=\rho\left(1+\rho+\rho^{2}+\rho^{3}+\rho^{4}+\rho^{5}+\cdots\right)
\end{gathered}
$$

Here, $\quad\left(1+\rho+\rho^{2}+\rho^{3}+\rho^{4}+\rho^{5}+\cdots\right)=\frac{1}{1-\rho} \quad$ is $\quad$ a convergent geometric series because previously we assumed that $\rho<1 . \Rightarrow S=\frac{\rho}{(1-\rho)^{2}}=\sum_{n=1}^{\infty} n \rho^{n}$

So, from equation (3) $L_{s}=(1-\rho) \sum_{n=0}^{\infty} n \rho^{n}$

$$
\Rightarrow L_{s}=(1-\rho) \sum_{n=0}^{\infty} n \rho^{n}=(1-\rho) \frac{\rho}{(1-\rho)^{2}}=\frac{\rho}{1-\rho}
$$

Where,

$$
\rho=\frac{\lambda}{\mu} \Rightarrow L_{s}=\frac{\rho}{1-\rho} \Rightarrow L_{s}=\frac{\lambda}{\mu-\lambda}
$$

Therefore, $L_{s}$ in terms of $\lambda$ and $\mu$ could be

$$
L_{s}=\frac{\lambda}{\mu-\lambda}
$$

When we observe equation (5), similar to the previous analogy (see equation (4)), $\lambda$ should be less than $\mu$ that is $\lambda<\mu$. Otherwise $L_{s}$ is negative which is meaningless or does not show the physical meaning of the reality. Since the number of vehicles waiting in the system $\left(L_{S}\right)$ is derived in terms of $\lambda$ and $\mu$ as shown in equation (5), the remaining steady state measures of performance $\left(W_{s}, W_{q}, L_{q}\right.$ etc) could be derived by combining equation (5) and Theorem 1 . So, first let us see Little's queuing formula shown in Theorem 1.

Theorem 1: For any queuing system in which a steadystate distribution exists, the following relations hold:

$$
\begin{aligned}
& L_{s}=\lambda W_{s} \\
& L_{q}=\lambda W_{q} \\
& L_{r}=\lambda W_{r}
\end{aligned}
$$

Our interest is finding the average amount of time a typical vehicle spends in a queue $\left(W_{q}\right)$ to use a service facility (road intersection) and the average time taken to be serviced or service time $\left(W_{r}\right)$ to leave the road intersections. By definition, average service rate $(\mu)$ and average service time $\left(W_{r}\right)$ can be determined using:

$$
W_{r}=\frac{1}{\mu}
$$

Here $\mu$ could be determined using equation (11).

And using equation (5) and theorem 1 we have:

$$
W_{s}=\frac{1}{\mu-\lambda}
$$

By definition:

$$
W_{s}=W_{q}+W_{r}
$$

and

$$
L_{s}=L_{q}+L_{r}
$$

By combining equation (6), equation (7) and equation (8) we have $W_{q}$ in-terms of $\lambda$ and $\mu$ : 


$$
W_{q}=\frac{\lambda}{\mu(\mu-\lambda)}
$$

Since the service rate $(\mu)$ specifies the average number of vehicles that can be serviced during a time period, it could be approximately determined according to the average arrival rate $(\lambda)$ and the average waiting time in queue $\left(W_{q}\right)$. Moreover the average waiting time in queue $\left(W_{q}\right)$ is known using Theorem 1, i.e., $W_{q}=\frac{L_{q}}{\lambda}$, thus the service rate $(\mu)$ could be calculated using equation (10).

So,

$$
\begin{gathered}
\Rightarrow W_{q}=\frac{\lambda}{\mu(\mu-\lambda)} \Rightarrow W_{q}\left(\mu^{2}-\mu \lambda\right)=\lambda \\
\Rightarrow W_{q}\left(\mu^{2}-\mu \lambda\right)-\lambda=0
\end{gathered}
$$

Hence, the only unknown variable is $\mu$ and the equation is quadratic. Therefore, $\mu$ can be solved using the equation:

$$
\mu=\frac{\lambda W_{q} \pm \sqrt{\left(\lambda W_{q}\right)^{2}+4 W_{q} \lambda}}{2 W_{q}}
$$

Traffic signals operate in a system of cross road intersections. Although a complete discussion was not include, signalized intersection has a lot of terminologies that must be known before analysis. Some of them are; the concept of phasing or signal timing (phase is the part of the cycle assigned to a fixed set of traffic movements), cycle (complete sequence of intervals), cycle length (the time it takes to complete one cycle) and so on. Typically, traffic signals operate in one of three different control models (signal timing) [3]:

- Pre-time operation (fixed time control),

- Semi-actuated operation and

- Fully actuated operation.

In the pre-time operation case of the traffic signal, the cycle length, phases, and all intervals are preset (fixed). Each cycle of the signal is exactly like another and all intervals and phase times are the same. In the semi-actuated operation, detectors are placed on minor approaches to the intersection. In the fully actuated case, every intersection approach has a detector or detectors, and both cycle length and the green-light time for every phase of the intersection can be varied. In this thesis pre-time operation is used to simulate the result. The light was assumed red for 40 seconds, green for 20 seconds and yellow time was assumed 2 seconds [3].

Clearly the capacity of roundabouts and cross intersections is limited and can accommodate up to the critical arrival rates for both road intersections. As the arrival rate increases up to the critical arrival rate, throughput (number of vehicles being serviced) also increases up to the maximum throughput. Throughput remains constant if the arrivals of vehicles that pass through the junction are above the critical arrival rate. Hence, in section 3, for cost analysis purpose we focused up to critical arrival rates which implies up to maximum average waiting time of vehicles in the system (road intersection). So, in the coming section (section 3) we compare the average waiting time $\left(W_{S}\right)$ and number of vehicles in the system $\left(L_{S}\right)$ for roundabout and cross intersection and finally we quantify it in to monetary values for both types of road intersections.

\section{Result and Discussion}

\subsection{Comparison of Waiting Time for Roundabout and Cross Intersection}

If we assume that the arrival of vehicles in the road intersection is described by a Poisson process with parameter $\lambda$, then there exists a critical value $\lambda_{c}([3],[26])$, such that

- if $\lambda>\lambda_{c}$, the queue increases without limit, but

- if $\lambda<\lambda_{c}$, the queue remains finite with probability 1 .

Thus, as the arrival rate increases, the waiting time also increase up to the critical arrival rate. Above the critical arrival rate, the operational performance of road intersections will likely stop its function rapidly, large queue begins to build and the waiting time increases exponentially. This is because of the maximum capacity of road is limited. In other words whatever the arrival rate it may be, the number of vehicles that will enter to the road intersections is limited to certain approximated value. This value, for any road intersections, is the maximum number of vehicles to be serviced per unit time (maximum service rate) which is determined, here in this thesis, in our case using critical arrival rate.

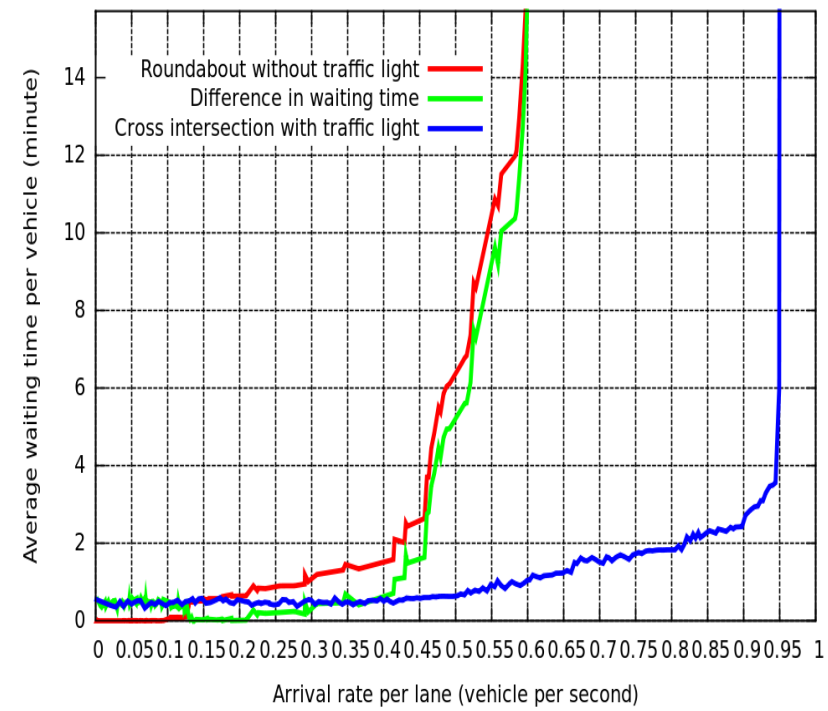

Figure 3. This figure shows the comparison of waiting time versus arrival rate between double lane roundabout without traffic light and double lane cross intersections with traffic light.

The number of vehicles serviced per unit time (service rate) depends on the number of vehicles arrived (arrival rate) to the road intersections and hence the service rate $(\mu)$ is simulated using equation (10). And consequently average waiting time in the system $\left(W_{s}\right)$ can be determined using equation (6) and the simulated result is seen from figure 3. As it can be shown from Figure 3, the critical arrival rate 
$\left(\lambda_{c}\right)$ for a roundabout and a cross intersection vary.

The critical arrival rate $\left(\lambda_{c}\right)$ for a roundabout is estimated to be 0.6 vps [3]. When the arrival rate is approximately greater than this value, the number of vehicles waiting in the system increases rapidly implying that flow of vehicles that utilize the road junction eventually saturated. So, the simulated result shows that relatively normal traffic flow was only up to the critical arrival rate.

\subsection{Cost Models}

Number of vehicles waiting too long time at road intersections could result in high cost to the drivers and passengers. The time wasted on the queue would have been wisely utilized elsewhere if the time was not lost what economists call it opportunity cost of time spent in queuing. Since waiting time and number of vehicles waiting at the system is known, quantifying it to cost is the next task. We have to find the time lost for passengers and extra fuel consumption by vehicles and quantifying it to monetary value (cost analysis). The maximum average waiting time $\left(W_{s}\right)$ for each vehicle at roundabouts and cross intersections respectively are approximated to $W_{s}=15.708 \mathrm{~min}(0.2618$ hr) and $W_{s}=5.971 \mathrm{~min}(0.099 \mathrm{hr})$ (see Figure 3). If vehicles wait more than these time, the queue builds exponentially and the road junctions may stop its proper function at all.

From Figure 3, the difference in waiting time of a roundabout and cross intersection calculated was based on the arrival rate $(\lambda \leq 0.6 v p s)$. As we discussed in the previous sections, if the arrival rate is greater than $0.6 \mathrm{vps}$, the difference in waiting time increases exponentially. Therefore, the average waiting time $\left(W_{d}\right)$ was calculated with the arrival rate up to $0.6 v p s$ (see Figure 3). Average waiting time of vehicles at roundabout $\left(W_{s r}\right)$ and average waiting time of vehicles at cross intersection $\left(W_{s c}\right)$ was determined from figure 3 . With $\lambda \leq 0.1$ vps vehicles waiting time at cross intersection is greater than roundabouts. i.e., $W_{s r}<W_{s c}$. So, we used the absolute value as the waiting time cannot be negative. Hence, to estimate the costs using the difference in waiting time and the difference in number of vehicles, we have to classify the ranges of $\lambda$. For $\lambda \leq 0.1$ vps the difference in waiting time incurred cost at cross intersection. Whereas for 0.1 vps $\leq$ $\lambda \leq 0.6 v p s$, the difference in waiting time incurred cost at roundabout.

$$
W_{d}=\left|W_{s r}-W_{s c}\right|
$$

where:

$W_{d}$ the difference in average waiting time

$W_{s r}$ average waiting time of vehicles at roundabout

$W_{s c}$ average waiting time of vehicles at cross intersection.

In the same way, number of vehicles $\left(L_{S}\right)$ waiting at roundabout and cross intersection is estimated using equation (4). So, it is possible to approximate the difference in number of vehicles waiting at road junctions $\left(L_{d}\right)$. As we discussed in the previous section, to compare the average waiting time of roundabout and cross intersection we only used up to the critical arrival rate of roundabout $(\lambda \leq 0.6$ vps $)$.
Correspondingly, we can estimate the difference in number of vehicles $\left(L_{d}\right)$ waiting at road junctions. Thus, the difference in number of vehicles $\left(L_{d}\right)$ waiting could be calculated using:

$$
L_{d}=\left|L_{s r}-L_{s c}\right|
$$

where:

$L_{d}$ the difference in number of vehicles waiting

$L_{s r}$ number of vehicles waiting at roundabout

$S_{s c}$ number of vehicles waiting at cross intersection.

Vehicles waiting or queuing at the road junction have mainly two cost components due to delay (congestion) although there are other costs like emission cost, psychological cost, and accident cost etc.

Major costs that we discussed in this research are:

- Cost of extra fuel consumed by vehicles

- Cost of lost time by passengers and drivers

\subsubsection{Cost of Extra Fuel Consumed Due to Waiting at the Road Junction}

To calculate the cost of extra fuel consumed by vehicles we need:

I. The difference in waiting time $\left(W_{d}\right)$ at the road junction.

II. The difference in number of vehicles $\left(L_{d}\right)$ waiting at the junction.

III. Average fuel consumption of a single vehicle per hour which is approximately estimated 1.263 litre per vehicle.

IV. Unit cost of fuel (assumed about 19 ETB).

Therefore, Expected Cost of Extra Fuel Consumed (ECEFC) per hour for a road junction is in the form of linear function:

$$
E C E F C=C_{u} \times F_{c} \times W_{d} \times L_{d}
$$

Where:

ECEFC Expected Cost of Extra Fuel Consumed per hour

$C_{u}$ Unit cost of fuel (19 ETB)

$F_{c}$ Extra fuel consumed for one vehicle per hour (1.263 litre per vehicle)

$W_{d}$ Difference in average waiting time at the road junction

$L_{d}$ the difference in number of vehicles waiting at the road junction.

Thus, Cost of annual extra fuel consumed due to congestion can be calculated as:

$$
A C E F C=E C E F C \times A C F
$$

Where:

$A C E F C$ Annual cost of extra fuel consumed

$E C E F C$ Expected Cost of Extra Fuel Consumed per hour

ACF Annual Conversion Factor (1300).

The cost of fuel lost is rapidly increase if vehicles are waiting much time or for $\lambda \geq 0.1 v p s$ and the cost incurred is at roundabout. For example, as it can be shown in Figure 4, the annual cost of extra fuel consumed due to delay was approximately estimated 560,000 ETB if vehicles are waiting about 6 minutes. 


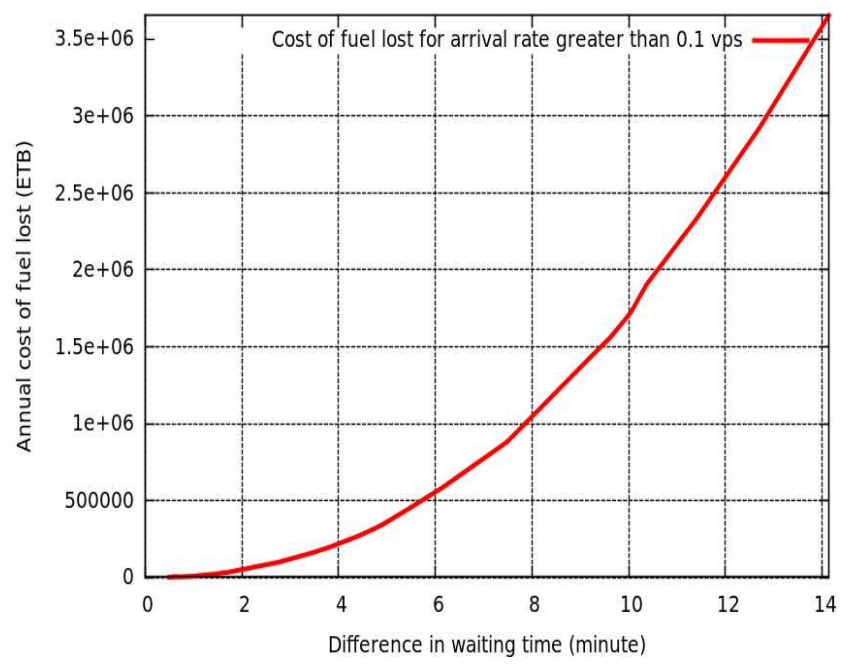

Figure 4. This figure shows the relationship between annual cost of extra fuel consumed by vehicles and waiting time at roundabout for 0.1 vps $\leq$ $\lambda \leq 0.6$ vps.

\subsubsection{Cost of Time Lost due to Waiting at the Road Intersection}

The delay cost of vehicles waiting at each road intersections due to congestion is not only the cost of extra fuel consumed but also it is an estimate of the value of time lost. The value of time lost is an opportunity cost which is caused by the effect of drivers and passengers waiting at road junctions. Passengers and drivers time lost at congested road intersections may be lost of working time or non working time to them when congestion is worsening.

Working time is the period of time that an individual spends at paid occupational labor. We assumed that, to convert the time lost (non productive time) to productive time the value of individual's working time is based on the average wage rate paid. The value of working time lost is an opportunity cost of time that passengers' waiting, total cost of the passenger's time, at each road intersections which is equal to the wage of individual passengers. On the other hand the value of non working time (off the job) is also an opportunity cost of time for passengers' that they may not be going to work rather the time to do something else and may be leisure time.

The basic thing is calculating the time lost of vehicles at each road junctions that is already determined in the previous sections. Clearly, the value of time lost varies from person to person. For instance, among those passengers some of them may be business men, intellectuals and in this case time is highly influential and for other passengers the value of time lost may not crucial. Therefore, passengers' wasting time by waiting some time at each road junctions loses their working time which reduces the productivity of the city. In anyway, whether passengers lost their working time or not, directly or indirectly the city's development is hindered.

Based on the value of time, the 2010 urban mobility report estimated the hourly value of time for a person in the US was estimated to be $\$ 16.30$ per hour [22]. But in Addis Ababa, Ethiopia, the average value of time for a working person and non-working could not be readily available when this research was conducted. Therefore, for simplicity we assumed that the value of time would be estimated, in this thesis, by converting the monthly average wage rate to hourly average wage rate and hence estimation of hourly income for an individual would be 11 ETB (Ethiopian Birr).

Other parameter which helps us to determine the cost of time lost is vehicle occupancy. We got the average vehicle occupancy of the Addis Ababa city traffic flow at major road intersections from [28] and the weighted average number of passengers in each vehicle was about $\left(V_{p}=10.9\right.$ passengers per vehicle).

Hence, the cost of time lost due to vehicles delay at the road intersection could be calculated as:

$$
C_{T}=L_{d} \times W_{d} \times t_{a} \times V_{p}
$$

Where:

$C_{T}$ Cost of time lost per hour

$L_{d}$ Difference in number of vehicles waiting in the system

$W_{d}$ Difference in average waiting time per vehicle

$t_{a}$ average value of time (11 ETB)

$V_{p}$ vehicle occupancy $\left(V_{p}=10.9\right)$

Hence, this equation can be used to find the cost of time lost at the road junction within one hour time. Moreover, we should convert it in to annual cost of time by using annual conversion factor. Thus, annual cost of time lost due to congestion can be determined by using:

$$
A C T L=C_{T} \times A C F
$$

Where:

ACTL Annual cost of time lost

$C_{T}$ Cost of time lost per hour

ACF Annual Conversion Factor (1300)

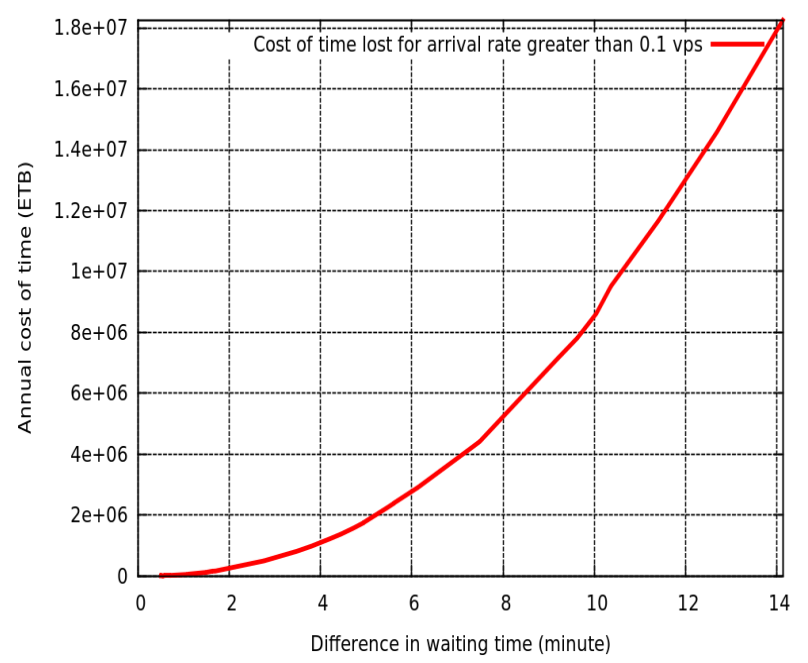

Figure 5. This figure shows the relationship between annual cost of time lost and difference in waiting time. The cost incurred is at roundabout since 0.1 vps $\leq \lambda \leq 0.6$ vps.

Figure 5, shows the relation between difference in waiting time and the annual cost of time lost. The cost lost increase as vehicles are waiting more time at the road junctions. For instance, vehicles waiting about 6 minutes at the junction 
costs passengers time lost which was approximately estimated 2,900,000 ETB per year.

Therefore, costs of time lost were estimated depending on the waiting time of vehicles at road junctions.

\subsubsection{Cost Comparison of Fuel and Time Lost}

From figure 6, we can conclude that cost of time lost is greater than cost of fuel lost when vehicles are waiting at the road junctions.

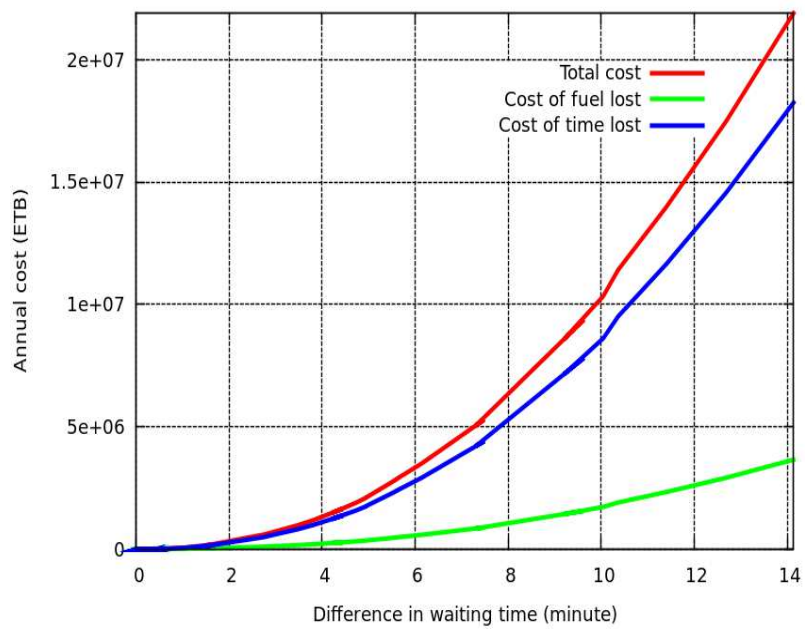

Figure 6. This figure shows Cost Comparison of Fuel and Time Lost.

\section{Conclusion and Recommendation}

\subsection{Conclusion}

- MMAS cellular automata and Poisson queuing model were used to simulate the average waiting time of vehicles.

- The maximum average waiting time of vehicles at a roundabout and cross intersection is $0.2618 \mathrm{hr}$ and $0.0995 \mathrm{hr}$ respectively.

- The cost of extra fuel consumed and cost of time lost for a roundabout and cross intersection are estimated according to the average waiting time.

- Much cost of time and fuel lost at roundabout than cross intersection.

\subsection{Recommendation}

- Further studies should be conduct including additional cost components like accident cost, construction and maintenance costs etc.

\section{Acknowledgements}

First, I would like to thank Dr. Semu Mitiku for his most support and encouragement. He kindly read my paper and offered invaluable detailed advices on organization and the theme of the paper. This research paper is made possible through the help and support of him.

Second, I would like to thank Mr. Yadeta Chimdessa who encouraged me and gave data that was useful for this research work

Third, I would like to thank Arba Minch University and Addis Ababa University who is financially helped me.

\section{References}

[1] H. J. Ruskin and R. Wang, "Modelling traffic Flow at Multilane Urban Round abouts", International Journal of Modern Physics C, Vol. 17 (2006)693-710.

[2] R. Kakooza, L.S. Luboobi and J.Y. T. Mugisha, , "Modelling Traffic Flow and Management at Signalized, Signalized and Roundabout Road intersections", Journal of Mathematics and Statistics, 1(2005), 194-202.

[3] Chimdessa Y. Seboka, Semu M. Kassa and Legesse Lemecha, "Efficiency of Roundabouts as Compared to Traffic Light Controlled Intersections in Urban Road Networks", Momona Ethiopian Journal of Science (MEJS), V5(2):81-100, 2013 CCNCS, Mekelle University, ISSN:2220-184X

[4] Robinson B. W., Rodegerdts L., Scarborough W., Kittelson W., Troutbeck R., Brilon W., Bondzion L., Courage K., Kyte M., Mason J., Flannery A., Myers E., Bunker J., Jacquemart G., "Roundabouts: an Informational Guide", U.S. Department of Transportation Federal Highway Administration, publication Number FHWA-RD-00-067, June 2000.

[5] Deo L., Akkawi F., Deo P., "Simulation model for urban ternary mix-traffic flow", Proc. of SPIE Vol. 6802,680210, (2008).

[6] Ding D., "Modeling and simulation of highway traffic using a cellular automaton approach", Department of Mathematics, Uppsala University,(December, 2011).

[7] Szklarski J., "Cellular automata model of self-organizing traffic control in urban networks", Bulletin of the Polish Academy of Sciences Technical Sciences, Vol. 58, No. 3, 2010, DOI: 10.2478/v10175-010-0041-3.

[8] Wang B. Eng., M. Eng., "Modeling Unsignalized Traffic Flow with Reference to Urban and Interurban Networks", School of Computer Applications, Dublin City University, (January 2003).

[9] Wallwork M.J, P.E., "Alternate Traffic Control: Roundabouts", Genesis Group, Inc., Jacksonville, Florida, (July, 2007).

[10] Furtado G., "Accommodating Vulnerable Road Users in Roundabout Design", A McElhanney Consulting Services Ltd., Surrey, BC, (August, 2007).

[11] Butch W., Metzer S.G., AICP, and Owens C.R., "Transportation Planning Division", American Planning Association, volume XXVII number 4 fall 2002.

[12] http://en.wikipedia.org/wiki/Roundabout, accessed on wikipedia, 06/05/2012.

[13] Zhang L., Matteis T. and Haubmann P., "Congestion Costs and Infrastructure Development: A Simulation Case Study", Institute for Economic Policy Research (IWW), Karlsruhe Institute of Technology, Waldhornstrasse 27, 6131.

[14] Hansen I., "Determination and valuation of Traffic Congestion Costs", EJTIR,1,no.1 (2001), pp. 61-72. 
[15] Das S., "A Queuing Analysis of Freeway Bottleneck Formation and Shockwave Propagation", M.Sc.Thesis, Civil Engineering, University of Minnesota, (July,1999)

[16] Minh C. C., Binh T. H., Mai T. T. and Sano K., "The Delay Estimation under Heterogeneous Traffic Conditions", Journal of the Eastern Asia Society for Transportation Studies, Vol.7, 2009.

[17] Chiguma M. L.M., "Analysis of Side Friction Impacts on Urban Road Links; Case study Dar-es-salaam", Stockholm, Sweden,ISSN 1653-4468, 2007.

[18] Pal M. and Sarkar D., "Delay, fuel loss and noise pollution during idling of vehicles at signalized intersection in Agartala city, India", Vol 2, No.6, 2012.

[19] H. J. Ruskin and R. Wang, "Modeling Traffic Flow at an Urban Unsignalized Intersection", ICCS 2002, LNCS 2329, Springer-Verlag Berlin Heidelberg, pp. 381390,2002.

[20] Shokri F., Mokhtarian H. R, Ismail A. and Rahmat R., "Comparing the Design of Roundabout and Intersection with aaSIDRA Software", European Journal of Scienti_c Research, ISSN 1450-216X Vol.40 No.2 (2010), pp.239-246.

[21] Vlahos E., Polus A. and Faghri A., "Comparative Analysis and Development of a Rating System for the conversion of AllWay Stop Controlled Intersecti into Roundabouts", Delaware Center for Transportation, Newark, Delaware 19716(302) 831-1446, (May, 2006)

[22] Schrank D., Lomax T. and Eisele B., "Texas Transportation Institute: Urban Mobality Reports", University Transportation Center for Mobility,(September, 2011).

[23] Taekratok T., "Modern Roundabouts for Oregon", Oregon Department of Transportation Research Unit, 200 Hawthorne SE, Suite B-240 Salem, OR 97310, June,1998).
[24] Taha H. A., "Operations Research: An Introduction, eighth edition", Pearson Education,Inc., 2007

[25] Hillier F. S. and Lieberman G. J., "Introduction to Operations Research, seventh edition", McGraw-Hill Companies, 2001

[26] Gazis D. C., "TraFFIc Theory ", Kluwer Academic Publishers, 2002

[27] Opara-Nadi G. E, "Electronic Selfcheckout System vs Cashier Operated System: a Performance Based on comparative Analysis ", PhD. Dissertation, School of Business,Capella University, (May, 2005)

[28] Taddesse W., "Assessing and Quantifying The Level of Traffic Congestion at Major Intersections in Addis Ababa", M.Sc.Thesis,Civil Engineering (Road and Transport Engineering) department,Addis Ababa University, (September, 2011)

[29] Lindsey C. R. and Verhoef E.T., "Congestion Modelling", Department of Economics,University of Alberta,(November, 1999)

[30] Wachs M., Samuels O. M., Skinner R.E., "Highway capacity Manual(HCM2000)",Transportation Research Board, National Research Council, published by the National Academy of Sciences (2000).

[31] Ngoc-Hien Do, Ngoc-Quynh-Lam Le, Ki-Chan Nam, "Coordination between traffic light system and traffic circle: A simulation analysis approach", International Journal of Mechanical Engineering and Applications Science Publishing Group, Vol. 3, No. 1-3, 2015, pp. 1-8.

[32] Güney Gorgun, Ibrahim Halil Guzelbey, "Simulation of traffic lights for green wave and dynamic change of signal", American Journal of Software Engineering and Applications Science Publishing Group, Vol. 2, No. 6, 2013, pp. 125-132. 